\title{
Aetiological considerations and risk factors for multi-infarct dementia
}

\author{
JOHN S MEYER, KAREN L MCCLINTIC, ROBERT L ROGERS, PENNE SIMS, \\ KARL F MORTEL
}

From the Cerebral Blood Flow Laboratory, Veterans Administration Medical Center, Department of Neurology, Baylor College of Medicine, and Neuropsychology Division, Department of Psychology, University of Houston, Houston, Texas, USA

SUMMARY One hundred and seventy five multi-infarct dementia (MID) patients were evaluated for risk factors for stroke as well as for the types of cerebrovascular lesions that were present. The incidence of associated risk factors for stroke were as follows: hypertension $(66 \%)$, heart disease $(47 \%)$, cigarette smoking $(37 \%)$, diabetes mellitus $(20 \%)$, moderate alcohol consumption $(19 \%)$ and hyperlipidaemia $(21 \%)$. The most frequently occurring type of lesions were multiple lacunar infarctions of the brain (43\%). These were combined with other types of stroke in an additional $21 \%$. Atherosclerotic occlusive disease of the carotid and vertebrobasilar arteries occurred alone in $18 \%$ and was associated with other types of stroke in another $25 \%$. Embolic cerebral infarctions were present alone in $8 \%$ and were combined with other types of stroke in $15 \%$. MID was more frequent in men $(62 \%)$ than women $(p<0.002)$. Mean bihemispheric gray matter cerebral blood flow (CBF) values showed a fluctuating course and when results were pooled and compared between different types of MID, extracranial occlusive disease and/or multiple lacunar infarctions resulted in lowest CBF values. The location of cerebral infarctions was more importantly related to cognitive impairments than was the total volume of infarcted brain. Mortality rates among 125 MID patients followed for 31 months has been $5 \%$. Correct clinical classification of the types of cerebrovascular lesions was confirmed in three necropsied cases.

Clinicopathological studies have shown that approximately $50 \%-65 \%$ of cases of dementia in the elderly are due to Alzheimer's disease (SDAT), $12 \%-20 \%$ are due to multi-infarct or vascular dementia (MID) and another $15 \%-20 \%$ exhibit neuropathological features of both SDAT and MID. ${ }^{1-2}$ Until 15 years ago, elderly dements with focal neurological signs were grouped together under the vague term "arteriosclerotic dementia". ${ }^{3-4}$ At that time, Hachinski and colleagues, ${ }^{5}$ basing their clinical investigations on earlier neuropathological studies, ${ }^{24}$ suggested the term "multi-infarct dementia" for patients with cognitive impairments resulting from multiple bilateral ischaemic strokes. They standardised a scale (Hachinski Index) for identifying patients with MID,

Address for reprint requests: J S Meyer, M.D., Director, Cerebral Blood Flow Laboratory, VA Medical Center, 2002 Holcombe Boulevard, Houston, Texas 77211, USA.

Received 15 December 1987 and in revised form 24 May 1988. Accepted 27 May 1988 based on the presence of hypertension, sudden onset of dementia, a fluctuating course and other characteristic features such as focal neurological symptoms and signs.

This Ischaemic Index has proven useful in differentiating MID from degenerative dementia of the Alzheimer type (DAT). ${ }^{56}$ Necropsy studies confirmed that, used alone, the Ischaemic Index is reliable for predicting the diagnosis of both MID and SDAT. ${ }^{78}$ It has a predictive accuracy of $71 \%$ for MID and $89 \%$ for SDAT. If the Ischaemic Index is combined with detailed medical, neurological and neuropsychological examinations, laboratory testing for risk factors for stroke, exclusion of other causes of dementia, and computed tomographic (CT) and/or magnetic resonance imaging (MRI) of the brain, clinical diagnostic accuracy is increased to $90 \%$. $^{39-13}$

In the study to be reported here, patients with MID were categorised into the different types of cerebral ischaemia and infarction which have been reported in the literature to give rise to MID, and into combina- 
tions of them if more than one type of stroke was present. Different types of stroke that may give rise to MID include multiple lacunar infarctions. ${ }^{4-14}$. The incidence of lacunar infarcts increases with age and is associated with risk factors for stroke, particularly hypertension. ${ }^{4913-15}$ Characteristic clinical features include abrupt onsets of cumulative neurological deficits, as described by Fisher, ${ }^{4}$ such as pure motor weakness, bradykinesis and rigidity, decreased spontaneous activity, dysarthria, small-stepped gait and later dementia. On CT and/or MRI imaging of patients with lacunes later confirmed at necropsy, small lesions have been present in the basal ganglia, thalamus, upper brain stem and white matter. Lacunar infarcts appear as small $(0.5-1.5 \mathrm{~cm})$ low density lesions by CT and as high density "bright objects" by $\mathrm{T}_{2}$ weighted MRI. They have a predominantly subcortical distribution consonant with neuropathological descriptions. ${ }^{3-59-14}$

Another type of MID results from the cumulative effects of multiple cerebral emboli. Embolic infarcts are usually larger than lacunes and usually have bilateral cerebral hemispheric distribution. Cerebral embolisation may also be confirmed during life by CT and/or MRI. Apart from their larger size, embolic lesions usually involve the cerebral cortex as well as subcortical white matter and are situated in the distributions of major cerebral arteries. Emboli causing MID may originate from a diseased heart, particularly if cardiac dysrhythmia is present, or from atherosclerotic plaques of the major aortocranial vessels, such as the internal carotid arteries and the vertebral arteries. $^{3913}$ Neurological deficits resulting from cerebral embolism are usually more pronounced than those arising from lacunar infarcts, and it is possible to predict which cerebral vessels have been occluded by the characteristic neurological features. The source of embolisation may be confirmed by aortocranial arteriography and/or ultrasonography if they arise from an atherosclerotic plaque of the major cerebral arteries and by echocardiography if they arise from a diseased heart.

Another type of MID results from the cumulative effects of multiple watershed or border zone infarctions, secondary to critical reductions of cerebral perfusion. 16-18 This has been termed "misery perfusion syndrome" by Samson, et al. ${ }^{18}$ based on positron emission tomographic (PET) measurements of severely reduced cerebral blood flow (CBF) with relatively well preserved cerebral metabolism in a series of 12 patients with severe occlusive disease of the carotid arteries who showed improvement of both blood flow and metabolism 3 weeks to 3 months after extra-intracranial arterial bypass procedures. Such patients with advanced bilateral atherosclerotic occlusive disease of the carotid and/or vertebral arte- ries may develop MID as a result of the reduced cerebral perfusion, a condition that may be confirmed during life by arteriography and/or Doppler ultrasonography. The characteristic distribution of the watershed infarcts of the brain can be identified by CT and/or MRI and the reduced cerebral perfusion may be measured by more readily available methods such as ${ }^{133} \mathrm{Xe}$ inhalation, as well as by PET scanning. ${ }^{61318}$

Rarer causes of MID include: inflammatory vasculitis associated with collagen vascular disease, progressive arteriosclerotic subcortical leucoencephalopathy, also termed Binswanger's disease, ${ }^{31319}$ ischaemia of the brain due to shunting of blood or "steal" into cerebral arteriovenous malformations, and vasospasm secondary to subarachnoid haemorrhage following rupture of an intracranial arterial aneurysm. ${ }^{3}$ Binswanger's disease is a chronic vascular dementia characterised neuropathologically by severe mural thickening of the small cerebral arterioles resulting in diffuse ischaemic gliosis of white matter and enlargement of the lateral ventricles, a condition almost invariably associated with hypertension. Clinically, Binswanger's disease is manifested by strokes, subacute accumulation of focal neurological symptoms and signs, plateau intervals followed by pseudobulbar palsy, dementia and prolonged motor deficits. ${ }^{19} \mathrm{CT}$ of the brain shows hydrocephalus ex vacuo with marked and diffuse attenuation of white matter. Binswanger's disease is identifiably different in aetiology from normal pressure hydrocephalus. ${ }^{20}$

There are several established risk factors that may enhance cerebral atherosclerosis, predispose to stroke, and/or reduce cerebral blood flow (CBF). ${ }^{1521-29}$ These include hypertension, diabetes mellitus, heart disease, hyperlipidaemia, cigarette smoking and alcohol consumption.

Hypertension has been identified as the single most important risk factor for both cardiovascular and different types of occlusive cerebrovascular disease, including MID $^{3031}$ transient ischaemic attacks (TIAs) $^{32}$, atheromatous occlusion or stenosis of the internal carotid and other major aortocranial arteries $^{33}$ and cardiogenic and arterio-arterial cerebral emboli. ${ }^{34}$ In the present protocol, subjects were considered to have a positive history for hypertension if its presence had been established in the past and antihypertensive medications had been prescribed or if there were repeated recordings of blood pressure in excess of $160 / 90 \mathrm{mmHg}$ on more than two consecutive occasions after adequate rest intervals. ${ }^{35}$

Cardiovascular disease more than doubles the risk of stroke in otherwise normal individuals, while among those that survive a stroke, it is the leading cause of death. ${ }^{36}$ In the present study, based on clinical and electrocardiographic evidence, patients with a 
diagnosis of arteriosclerotic heart disease with and without cardiac dysrhythmias and those with and without degenerative valvular disease, were included; however, patients with rheumatic heart disease were excluded.

Hyperlipidaemia has become widely accepted as a risk factor for atherosclerotic heart disease and has recently been implicated as a risk factor for cerebral atherothrombotic stroke and for cerebral atherogenesis, particularly of the larger cerebral vessels. ${ }^{37}$ In the present study, cholesterol and triglyceride levels were considered to be elevated when they exceeded normative values developed and published by the Methodist Hospital Lipid Clinic, Houston, Texas, ${ }^{38}$ where upper limits of normal for fasting values are 265 $\mathrm{mg} / \mathrm{dl}$ for cholesterol and $190 \mathrm{mg} / \mathrm{dl}$ for triglyceride levels.

Diabetes mellitus is well accepted as a risk factor for the development and progression of atherosclerosis of large and small cerebral vessels, ${ }^{39}$ and has been implicated as a substantial risk factor for atherothrombotic brain infarction, particularly among men. ${ }^{40}$ Diagnostic criteria used in the present protocol for diabetes mellitus were medical history of treatment for this condition plus documentation of hyperglycaemia and a satisfactory response to treatment by diet, oral hypoglycaemic agents or insulin. ${ }^{37}$

Rogers et $a l^{4142}$ have reported that alcohol consumption and habitual cigarette smoking both increase the risk for stroke, and that these increases in risk are directly related to the amount of each substance abused. For the purposes of the present study, patients were categorised according to the amount of alcohol consumed as follows: none $=$ never drinks alcohol; mild = less than 1-2 drinks per week; moderate = approximately 1-3 drinks per day; and those who quit at least 3 months prior to admission into the study. Heavy drinkers were excluded. Patients were also classified according to the amount of cigarettes they smoked: none $=$ never smoked; moderate $=$ less than one pack per day; heavy = more than one pack per day or smokes cigars or a pipe six or more times per day; and those who quit at least 3 months prior to admission into the study.

Prospective clinical trials, in which CBF measurements have been repeated at intervals among neurologically normal volunteers with risk factors for stroke before and after the development of strokes and MID, indicate that CBF is reduced for as long as 2 years prior to their onset. ${ }^{2425}$ Longitudinal clinical trials among patients with MID indicate that control of risk factors including control of hypertension, ${ }^{1322}$ cessation from cigarette smoking ${ }^{132829}$ and ventriculoperitoneal shunting procedures in some patients with associated normal pressure hydrocephalus, ${ }^{20}$ result in measurable improvements in
CBF and cognition.

The present clinical investigation was designed to explore the demography and frequency of distribution of the different types of MID and associated risk factors for stroke. The diagnoses of MID were confirmed in each patient by the clinical investigations reviewed above among 175 MID patients, 125 of whom agreed to be followed longitudinally. Age, sex, aetiological classifications and associated risk factors were determined and all of these variables were correlated with serial measurements of $\mathrm{CBF}$ values.

\section{Methods}

One hundred and seventy five consecutive patients with MID were admitted to the study. All were referred for diagnosis and treatment and were examined and evaluated according to a standard protocol. Patients referred included both veterans $(40 \%)$ and non-veterans $(60 \%)$. They were almost all referred as out-patients by neurologists, neurosurgeons, cardiovascular surgeons, and physicians from the Southwestern United States. They are considered to be a representative sample of patients with MID from this area. They were all asked to participate in longitudinal studies carried out on an out-patient basis and 125 of them agreed to do so.

To provide a comparative descriptive profile with the MID subjects, 125 neurologically normal, age-matched volunteers were recruited. These subjects are part of an on-going longitudinal investigation into the natural history of aging and cerebrovascular disease.

At the time of admission to the study, each patient underwent evaluation including (1) medical and neurological examinations, (2) Cognitive Capacity Screening Examinations (CCSE), ${ }^{13}$ (3) modified Hachinski Ischaemic Index, ${ }^{13}$ (4) regional cerebral blood flow (CBF) measurements using the ${ }^{133} \mathrm{Xe}$ inhalation method, ${ }^{43}{ }^{44}$ (5) blood chemistries, (6) electroencephalogram, and (7) computed tomography (CT) and/or magnetic resonance imaging (MRI) of the brain. A questionnaire was completed by the patients, assisted by their family if necessary, concerning demographic information, medications, and medical history. Diagnosis of MID was based on an Ischaemic Index of 5 or more, ${ }^{37-913}$ a CCSE score of 25 or less, ${ }^{13}$ history of sudden onset of stepwise and progressive mental deterioration, focal neurological symptoms and signs, abnormal brain imaging compatible with ischaemic lesions and patchy bilateral and asymmetric reductions of gray matter regional CBF.

Patients and their families who agreed to participate in the longitudinal study of multi-infarct dementia returned to the laboratory at intervals of 3-6 months. To date, 125 patients have agreed to participate and have been followed for an average of 31.3 , SD 26.8 months. The reason for the large standard deviation is that some patients have recently joined the study while others have been followed for as long as 9 years, so that the follow-up has varied between 3-116 months. At each visit, medical and neurological examinations, CBF measurements and CCSE testing were repeated.

The presence alone or in combination of the following aetiological causes for MID were identified for each patient: (1) Embolic infarctions were considered to be present among 
patients who had clinical and CT and/or MRI confirmation of non-haemorrhagic lesions within the distribution of the major cerebral arteries and/or evidence of extensive cortical infarction; (2) Lacunar infarctions were considered to be present among patients with a history and neurological signs and symptoms compatible with subcortical lacunar strokes $^{414}$ and/or identification of subcortical infarctions by CT and/or MRI. Circumscribed subcortical gray matter lesions and focal asymmetrical periventricular white matter lesions measuring $0.5-1.5 \mathrm{~cm}$ in greatest diameter were considered evidence of lacunar infarctions: ${ }^{10-14}(3)$ Misery perfusion with infarction was considered to be present in MID patients with angiographic evidence of severe stenosis or occlusion of the internal carotid arteries and/or the vertebral and basilar arteries, plus clinical, CT or MRI evidence of cortical and/or white matter lesions in the distribution of the watershed areas between the major cerebral arteries. ${ }^{16-18}$ The diagnosis of MID due to Binswanger's disease or vasculitis was based on standard clinical, CT and MRI findings, plus the usual laboratory diagnostic criteria that have been described for these disorders. ${ }^{319}$ Patients considered to have cardiogenic embolic strokes underwent echocardiography as well as electrocardiography in order to evaluate heart disease as their cause.

Procedures for measuring CBF were carried out according to methods described by Obrist et al. ${ }^{43}$ and modified for clinical use by Meyer et al. ${ }^{44}$ Briefly, five to eight millicuries of ${ }^{133} \mathrm{Xe}$ gas were mixed with room air and inhaled for one minute. Isotopic clearance throughout the ensuing 10 minutes was monitored by means of 16 collimated sodium iodide crystal detectors distributed symmetrically over each hemisphere. After correction for arterial recirculation, the fast component of clearance was used to estimate gray matter flow. ${ }^{43}$ Pulse rate, end-tidal $\mathrm{PCO}_{2}$, respiratory rate, and EEG were monitored throughout.

Protocols for these studies have been approved by the Institutional Review Boards of VA Medical Center, Houston and Baylor College of Medicine and are reapproved annually by the VA Medical Center.

\section{Statistical analyses}

The Chi-square statistic was used to determine frequency distributions for age, gender, risk factors and for different aetiological factors considered responsible for cerebral ischaemia or stroke among the entire case series. Sets of one-way analysis of variance (ANOVA) were employed to ascertain any relationships between group comparisons of initial, mean, lowest and highest CBF values, and to determine any age differences between different diagnostic groups. Significant results were followed by pairwise contrasts to compare CBF values among different groups. In addition, analysis of covariance was used to determine if any significant age differences existed between diagnostic groups and to determine if age, which is a risk factor for stroke itself, influenced results.

\section{Results}

As shown in table 1, the MID group as a whole had an average age of $67 \cdot 1$, SD $10 \cdot 2$ years and $62 \%$ were male. Table 1 summarises results of analyses for all
Table 1 Patients with MID

\begin{tabular}{lc}
\hline TOTAL NUMBER & 175 \\
Mean Age & $67 \cdot 1$, SD $10 \cdot 2$ \\
Mean Ischaemic Index & $8 \cdot 1$, SD $1 \cdot 8$ \\
Males/Females & $61 \cdot 7 \% / 38 \cdot 3 \%$ \\
Associated SDAT (MIX) & $6 \cdot 5 \%$ \\
Mean Initial Visit CBF* & $60 \cdot 6+$, SD $10 \cdot 1$ \\
Age Matched Mean Normative CBF & $68 \cdot 4$, SD 9.1 \\
TOTAL NUMBER RETURNING FOR & \\
SERIAL VISITS & 125 \\
Mean Follow-up Interval & $31 \cdot 3$, SD $26 \cdot 8$ \\
Mean CBF* Over-all Visits & $61 \cdot 0$, SD 8.1 \\
Lowest CBF* at Any Visit & $55 \cdot 5$, SD 9.0 \\
Highest CBF* at Any Visit & $66 \cdot 5$, SD $10 \cdot 8$ \\
\hline
\end{tabular}

*Mean bihemispheric gray matter blood flow in $\mathrm{mls} / 100 \mathrm{gm}$ brain/minute

tSignificantly reduced compared with age matched normals $(\mathrm{p}<0.0005)$.

subjects according to mean age, gender, ischaemic indices, mean interval of follow-up and initial CBF values. As shown in table 1, of the total of 175 MID cases, $6.5 \%$ were considered to show, in addition to MID, some clinical symptoms and/or neuroimaging features characteristic of associated SDAT and were classified as having a mixture of both MID and SDAT (MIX).

Table 2 shows the frequencies of occurrence for associated risk factors for stroke among all the MID and neurologically normal cases. The case series of MID patients included 162 caucasians, nine blacks, two hispanics, and two Asian-Americans. Chi-square analysis was used to test for significance of gender distribution. Men accounted for $62 \%$ of MID cases which was significantly greater than representation for women $(p<0.002)$. As seen in table 2, Chi-square analysis of frequency distributions showed significant differences between the MID group and the neurologically normal volunteers in the occurrence of hypertension $(p<0.0002)$, heart disease $(p<0.0026)$, diabetes mellitus $(p<0.0027)$, cigarette smoking $(\mathrm{p}<0.0001)$, and carotid bruits $(\mathrm{p}<0.007)$.

Table 3 summarises the frequency of occurrence of different causes of stroke and cerebral ischaemia among the MID patients. In the present series of patients, subcortical lacunar infarctions were the most frequently occurring cause of MID. These were present alone in $43 \%$ of the MID patients and were associated with misery perfusion in $13 \%$ and with major embolic strokes in $4.0 \%$ of the series. Clinical evidence of subcortical lacunar infarctions, associated with focal gray and white matter lesions of $0.5-1.5 \mathrm{~cm}$ in diameter demonstrated by CT and MRI imaging, was present in a total of $65 \%$ of the present series of MID patients.

The second most frequent aetiology of MID was misery perfusion due to severe extracranial atherosclerosis with occlusion or stenosis of the carotid and/or the vertebral arteries. This was the sole cause 
Table 2 Associated risk factors

\begin{tabular}{|c|c|c|c|c|c|}
\hline \multirow[b]{2}{*}{ Risk Factors: } & \multicolumn{2}{|c|}{ Mid subjects } & \multicolumn{2}{|c|}{ Normal controls } & \multirow[b]{2}{*}{$\begin{array}{l}\text { Level of* } \\
\text { Significance }\end{array}$} \\
\hline & $\begin{array}{l}\text { Case } \\
\text { Numbers }\end{array}$ & $\begin{array}{l}\text { Percentage } \\
\text { Frequency }\end{array}$ & $\begin{array}{l}\text { Case } \\
\text { Numbers }\end{array}$ & $\begin{array}{l}\text { Percentage } \\
\text { Frequency }\end{array}$ & \\
\hline $\begin{array}{l}\text { Hypertension } \\
\text { Heart Disease } \\
\text { Diabetes mellitus } \\
\text { Hyperlipidaemia }\end{array}$ & $\begin{array}{r}116 \\
83 \\
35 \\
36\end{array}$ & $\begin{array}{l}66 \cdot 3 \% \\
47 \cdot 4 \% \\
20 \cdot 0 \% \\
20 \cdot 6 \%\end{array}$ & $\begin{array}{r}55 \\
36 \\
9 \\
25\end{array}$ & $\begin{array}{r}44 \cdot 0 \% \\
28 \cdot 8 \% \\
7 \cdot 2 \% \\
20 \cdot 3 \%\end{array}$ & $\begin{array}{l}\mathrm{p}<0.0002 \\
\mathrm{p}<0.0026 \\
\mathrm{p}<0.0027 \\
\mathrm{NS}\end{array}$ \\
\hline $\begin{array}{l}\text { Cigarette Smoking } \\
\text { None } \\
\text { Moderate } \\
\text { Heavy } \\
\text { Former Smokers Who Quit }\end{array}$ & $\begin{array}{l}59 \\
21 \\
36 \\
58\end{array}$ & $\begin{array}{l}33 \cdot 7 \% \\
12 \cdot 0 \% \\
20 \cdot 6 \% \\
33 \cdot 1 \%\end{array}$ & $\begin{array}{r}72 \\
7 \\
10 \\
34\end{array}$ & $\begin{array}{r}58 \cdot 5 \% \\
5.7 \% \\
8 \cdot 1 \% \\
27 \cdot 6 \%\end{array}$ & $\mathrm{p}<0.0001$ \\
\hline $\begin{array}{l}\text { Alcohol Consumption } \\
\text { None } \\
\text { Mild } \\
\text { Moderate } \\
\text { Former Drinkers Who Quit }\end{array}$ & $\begin{array}{l}64 \\
65 \\
33 \\
13\end{array}$ & $\begin{array}{r}36 \cdot 6 \% \\
37 \cdot 1 \% \\
18 \cdot 9 \% \\
7 \cdot 4 \%\end{array}$ & $\begin{array}{r}32 \\
50 \\
24 \\
6\end{array}$ & $\begin{array}{r}28 \cdot 6 \% \\
44.6 \% \\
21.9 \% \\
5 \cdot 4 \%\end{array}$ & NS \\
\hline Carotid Bruits & 20 & $11.4 \%$ & 3 & $2.4 \%$ & $p<0.0070$ \\
\hline
\end{tabular}

*Chi-square analysis of frequency distributions

of MID in $18 \%$ and was associated with other causes of stroke in $25 \%$.

The third most frequent cause was multiple major embolic strokes $(8.0 \%)$. Embolic strokes were combined with other types of stroke in an additional $15 \%$. Mixtures of two or more different types of stroke were combined among $29 \%$ of MID patients. Of all the MID patients suffering from multiple embolic strokes, $24 \%$ had an identifiable cardiogenic source for emboli.

As shown in table 1, mean values for bihemispheric gray matter CBF at the initial visit among the MID patients were significantly reduced compared with age-matched normal values $(p<0.0005)$. Mean CBF values were compared between the three MID groups categorised as due to misery perfusion syndrome, multiple embolic strokes, and multiple lacunar infarctions. For these analyses, patients with combined causes and those with rare aetiologies of MID such as Binswanger's disease and vasculitis, were excluded. Three measures of CBF collected from the patients who agreed to longitudinal CBF measurements were subjected to analysis of variance (ANOVA). These included mean CBF values calculated from all visits, and the lowest and highest CBF

Table 3 Aetiological classification

\begin{tabular}{lcc}
\hline Etiology & Number & Frequency (\%) \\
\hline Lacunar infarcts & 76 & $43 \cdot 4$ \\
Misery perfusion (M.P.) & 32 & $18 \cdot 3$ \\
M.P. + lacunar infarcts & 23 & $13 \cdot 1$ \\
Major embolic strokes + M.P. & 13 & $7 \cdot 4$ \\
Major embolic strokes & 14 & $8 \cdot 0$ \\
Strokes + lacunar infarcts & 7 & $4 \cdot 0$ \\
M.P. + strokes + lacunar infarcts & 7 & $4 \cdot 0$ \\
Vasculitis & 2 & $1 \cdot 1$ \\
Binswanger's disease & 1 & $0 \cdot 6$ \\
TOTAL & 175 & $100 \cdot 0$ \\
\hline
\end{tabular}

values measured at any visit. The figure illustrates between group comparisons of CBF values for MID patients in the three groups with misery perfusion, or embolic or lacunar strokes. All groups showed marked fluctuations of $\mathrm{CBF}$ values at return visits, which exceed those seen in Alzheimer's disease. ${ }^{45-48}$ As shown in the fig, mean CBF values for the group with embolic strokes was significantly higher than for the group with misery perfusion $(p<0.02)$, or the group with multiple lacunar infarctions $(p<0.01)$. No significant differences were apparent when mean CBF values were compared between the group of patients with misery perfusion and the slightly older group suffering from multiple lacunar infarctions. Significant effects $(p<0.04)$ were also observed between the three principle causes of MID when lowest CBF values were compared $(p<0.04)$. There were significantly higher $\mathrm{CBF}$ values among the group with multiple embolic strokes compared with the group with multiple lacunar infarctions $(p<0.02)$. Between group analyses for the highest CBF levels followed similar patterns as those found for mean and for lowest CBF values. There were significantly lower CBF values among the group with misery perfusion compared to the group with embolic stroke $(\mathrm{p}<0.02)$.

Analysis of variance did not indicate significant differences for mean age between the three MID groups. However, the mean age for the group with multiple lacunar infarctions (70.3, SD 9.9) was slightly older than the groups with embolic strokes $(66 \cdot 0$, SD $13.2)$ or with misery perfusion $(65.9$, SD 8.7$)$. Since decreases of CBF are associated with aging, ${ }^{21}$ it was considered possible that even these slight differences in age might contribute to some of the group differences for CBF. Therefore, analyses were repeated using a covariance model to correct for agedependent relationships. After making this correction 


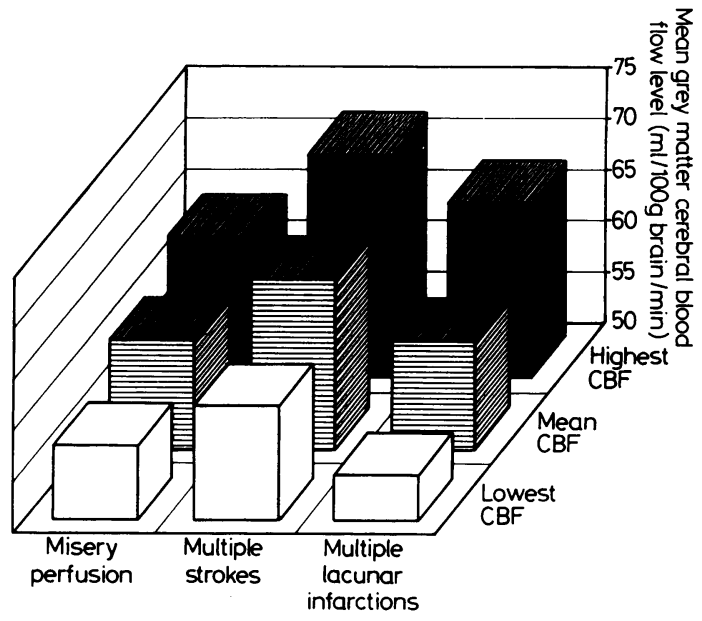

Fig 1 Mean bihemispheric gray matter $C B F$ values among the three major clinical categories of MID (subcortical lacunar infarcts, misery perfusion, and major embolic strokes) comparing mean, highest and lowest $C B F$ values for all serial $C B F$ measurements recorded during the prospective trial. As judged by all three CBF measures, $M I D$ patients with major embolic strokes had significantly higher CBF values than MID patients with misery perfusion or subcortical lacunar infarcts.

for differences in ages between groups, results were similar to those already described without correcting for age differences.

Among the 125 MID patients followed for 31 months the mortality rate has been $5 \cdot 1 \%$. Necropsy confirmation of the diagnosis of MID has been obtained in three patients and correct classification of the type of cerebral ischaemia and absence of microscopic features of SDAT was confirmed in all three. Results of the three necropsy examinations will be reviewed briefly. The first patient was a 45 year old male with insulin-dependent diabetes and hypertension. Clinically, he was classified as MID due to multiple lacunar infarctions. He died of acute pontine haemorrhage. At necropsy, there was advanced atherosclerosis of the cerebral vessels with marked stenosis of the basilar artery and multiple lacunar infarcts of the brain measuring $0.5-1.2 \mathrm{~cm}$ in diameter. These were located in the thalamus bilaterally, the left globus pallidus, the right putamen and the right dentate nucleus. The second patient was a 74 year old male with atherosclerotic heart disease and myocardial infarction complicated by cardiac arrhythmia. He was classified as MID due to multiple cardiogenic emboli. He died of heart failure. At necropsy there were multiple embolic infarcts of the brain secondary to coronary artery atherosclerosis as well as myocardial infarction which was considered to be the cause of the longstanding cardiac dysrhythmia during life. The embolic infarcts involved the entire right caudate nucleus and surrounding white matter of the frontal lobe, the left occipitotemporal cortex in the distribution of the posterior cerebral artery and the left parietal cortex in the distribution of the middle cerebral artery. The third patient had longstanding hypertension and was classified as MID due to misery perfusion. He died of arteriosclerotic heart disease with heart failure and complications following aortofemoral reconstructive surgery. At necropsy, there was complete, organised, thrombolic occlusion of the left internal carotid artery as well as severe atherosclerotic stenosis of the right intracranial internal carotid artery confirming the diagnosis made by cerebral arteriography during life. Several years earlier, he had undergone surgical by-pass of the left superficial temporal artery to the middle cerebral artery. The by-pass was patent but there were multiple recent and remote infarcts in the watershed territories between both middle cerebral arteries and the anterior and posterior cerebral arteries, most evident in the left central hemisphere. There were also lacunar infarctions present in the right cerebellum.

\section{Discussion}

Experience from this study suggests that MID is most commonly encountered after 60 years of age and affects predominantly males. MID is almost invariably associated with remediable risk factors for stroke and occurs in blacks, whites, Asian and Hispanic Americans. As judged by the present series, the most frequent cause of cerebral ischaemia and infarctions associated with MID are multiple lacunar infarcts. The second most common cause is severe extracranial occlusive disease of the carotid, vertebral and basilar arteries and the third most common cause is multiple cerebral emboli arising from the internal carotid arteries or from the heart. Many patients with MID are referred to this laboratory for evaluation of cerebral perfusion prior to consideration of surgical revascularisation procedures such as carotid endarterectomy and superficial temporal to middle cerebral artery by-pass. It is possible that more cases of extracranial occlusive disease were admitted to this study than would be encountered by other referral centres. Likewise, the majority of referrals to this laboratory are out-patients so that it is possible that the occurrence of MID due to multiple major cerebral arterial embolic occlusions may have been underestimated because severely ill patients hospitalised with stroke are often discharged to convalescent homes without completing the detailed investigations required by the research protocols of this laboratory.

Tomlinson et al. ${ }^{2}$ suggested that small lacunar 
infarcts are not as damaging to cognitive performance as multiple major strokes based on the observation that lacunar infarcts are frequently encountered at necropsy in the brains of the nondemented elderly. Present results are in agreement with those of Fisher ${ }^{4}$ who reported that multiple small lacunar infarcts may eventually become associated with mental deterioration. It is apparent, however, that lacunar strokes are frequently encountered on examining CT and MRI scans among elderly subjects both with and without dementia, and many of these strokes appear to have been clinically silent. ${ }^{10-121445}$

Among MID patients, those with multiple embolic strokes showed higher CBF values compared to those with extracranial occlusive disease or those with multiple lacunar infarcts. This is of interest since patients with major embolic strokes have larger volumes of infarcted brain tissue measured at necropsy or by CT or MRI during life, compared with those with lacunar infarcts or those with extracranial occlusive disease and watershed infarcts. This suggests that the severely reduced cerebral perfusion associated with extracranial occlusive disease or associated with multiple lacunar infarcts is not solely due to the brain infarctions but also reflects diffuse cerebral ischaemia secondary to occlusive disease of the large and small cerebral arteries. Among patients with unilateral carotid occlusion demonstrated by angiography, there are not only marked reductions in regional CBF of the ipsilateral hemisphere, but also of the contralateral hemisphere, due to interhemispheric steal. ${ }^{1318}$ Likewise, neuropathological studies of patients dying with multiple lacunar infarctions show widespread liphohyalinosis, mural thickening and stenosis of the cerebral arterioles including penetrating end-arteries supplying the subcortical white matter. ${ }^{4} 19$

It has been postulated from CBF measurements that otherwise healthy gray and white matter in patients with MID may become reversibly impaired by severely diminished blood supply and that this reversible ischaemia contributes to the cognitive impairments. ${ }^{184647}$ The fluctuating course, which is so characteristic of patients with MID, is best explained by intermittent ischaemia of gray and white matter without infarction. ${ }^{48}$ If cognitive declines among MID patients were solely due to the volume of infarcted brain tissue, fluctuations in the clinical course with subsequent improvements in CBF and cognition would be difficult to explain. This haemodynamic concept is supported by investigations showing that CBF and cognition fluctuate together in patients with MID. ${ }^{1348}$ If this haemodynamic concept proves to be correct, it may have important implications concerning prevention and treatment of MID.

The second most frequently occurring cause of MID, in the present series of MID patients, was misery perfusion due to severe atherosclerotic extracranial occlusion. This occurred alone in $18 \%$ but a comparable number had misery perfusion plus associated lacunar infarcts or associated embolic strokes or both (table 3 ). This should be borne in mind if surgical revascularisation procedures ${ }^{18}$ are considered in MID patients with severe extracranial occlusive disease, since surgical treatment is not likely to improve cognitive impairments due to embolic or lacunar infarcts, whereas medical treatment is more likely to do so. ${ }^{13}$

The present study confirms the higher frequency of occurrence of MID among males $(62 \%)$ than females. This preponderance of MID is considered to be valid, since non-veterans of either sex, as well as male and female veterans, are referred to this laboratory. A series of 28 patients with senile dementia of the Alzheimer's type referred to this laboratory show a female to male preponderance of $4: 3$, similar to ratios reported by other centres of large series of patients with SDAT. ${ }^{4950}$ In general, male to female ratios of between 1.5-2.1 have been reported among cohorts with either stroke or MID. ${ }^{4950}$ The higher incidence of risk factors for stroke that occurs among men, particularly hypertension and heart disease, most likely explains the preponderance of male patients with stroke and MID. ${ }^{51} 52$ Conversely, risk factors for stroke are infrequent among patients with pure Alzheimer's disease. Women survive longer than men and are more likely to suffer from Alzheimer's disease, since SDAT is also age-related. ${ }^{49}$

Hypertension is the most frequent vascular risk factor among patients with MID and occurred in $66 \%$ of the present series. This is to be expected since hypertension is the most potent risk factor for stroke. Epidemiological studies have shown that hypertension is associated with six times the risk for stroke compared with that among age-matched normotensive subjects. ${ }^{52}$ Heart disease is also a potent risk factor for MID and was present in $47 \%$ of the present series. Heart disease carries an increased risk for stroke 2 to 5 times that among age-matched subjects without heart disease. This increased risk for stroke associated with heart disease is not reduced by control of associated hypertension. ${ }^{52} \mathrm{~A}$ history of cigarette smoking was present in $33 \%$ of the present series of patients with MID, while $19 \%$ were considered to be moderate drinkers and each of $20 \%$ had associated diabetes mellitus or Type IV hyperlipidaemia. Multiple risk factors were encountered frequently and these individuals were considered to be at high risk for progressive dementia.

Until recently, relationships between cognition and CBF were difficult to elucidate because of the widely fluctuating course which is so characteristic of MID patients. Longitudinal studies, however, have shown that coupling does exist between the wide fluctuations 
of both CBF and cognition. ${ }^{48}$ Since diffuse cerebral ischaemia in risk factored subjects precedes the onset of strokes and MID, ${ }^{25}$ control of risk factors and prevention of transient ischaemic attacks may prevent or improve the cognitive deficits in this form of dementia among the elderly.

This work was supported by the Veterans Administration, Washington, D.C. and grants from Harry K. and Albert K. Smith; Deloitte, Haskins and Sells; the J. S. Abercrombie Foundation, and Entex, Inc., all of Houston.

\section{References}

1 Katzman R, Terry RD. The Neurology of Aging. Philadelphia, Davis Co. 1983; 1-14.

2 Tomlinson BE, Blessed GI, Roth M. Observations of brains of demented old people. J Neurol Sci 1970;11:205-42.

3 Loeb C. Vascular Dementias. In: Fredrecks JAM (ed.); Handbook of Clinical Neurology Vol. 2. Neurobehavioural Disorders. Amsterdam: Elsevier Science Publishers, 1985; pp. 353-369.

4 Fisher CM. Dementia in cerebral vascular disease. In: Toole JG, Siekert RG, Whisnant JP (eds), Cerebral Vascular Disease, Sixth Princeton Conference. New York, Grune and Stratton, 1968; 232-6.

5 Hachinski VC, Lassen NA, Marshall J. Multi-infarct dementia: a cause of mental deterioration in the elderly. Lancet 1974;ii:207-9.

6 Hachinski VC, Iliff L, Duboulay GH, et al. Cerebral blood flow in dementia. Arch Neurol 1975;32:632-7.

7 Rosen WG, Terry RD, Fuld PA. et al. Pathological verification of ischaemic score in differentiation of dementia. Ann Neurol 1980;7:486-8.

8 Molsa PK, Puljarvi L, Rinne J, et al. Validity of clinical diagnosis in dementia: a prospective clinicopathological study. J Neurol Neurosurg Psychiatry 1985;48:1085-90.

9 Loeb C, Gandolfo C, Bino G. Correlations beween cerebral lesion and the demented state in patients with multiple infarcts. In: Meyer JS, Lechner H, Reivich M, Ott E., Cerebrovascular Disease 6: Amsterdam, Excerpta Medica, The Netherlands, 1988, 123-8.

10 Rolinvock JF, Lydar PD, Hesselich JR, et al. Brain magnetic resonance imaging in the evaluation of lacunar stroke. Stroke 1987;18:781-6.

11 Erkinjuntti T, Ketonen L, Sulkava R. et al. Do white matter changes on MRI and CT differentiate vascular dementia from Alzheimer's disease. J Neurol Neurosurg Psychiatry 1987; 50:37-42.

12 Fazekas F, Chawluk JB, Alavi A, et al. MR signal abnormalities at $1.5 \mathrm{~T}$ in Alzheimer's dementia and normal aging. AJNR 1987;8:421-2.

13 Meyer JS, Judd BW, Tawaklna T, Rogers RL, Mortel KF. Improved cognition after control of risk factors for multiinfarct dementia. JAMA 1986;256:2203-9.

14 Bamford J, Sandercock P, Jones L, Warlow C. The natural history of lacunar infarction: the Oxfordshire community stroke project. Stroke 1987;18:545-51.

15 Dyken ML, Wolf PA, Barnett HJM, et al. Risk factors in stroke. A statement for physicians by the subcommittee on risk factors and stroke of the Stroke Council. Stroke 1984;15:1105-11.

16 Howard R, Trend PJ, Russell RWR. Clinical features of ischaemic lesions in cerebral arterial borderzones. Arch Neurol. 1987;44:934-9.

17 Bogousslavsky J, Regli F. Unilateral watershed cerebral infarcts. Neurology 1986;36:373-7.
18 Samson Y, Baron JC, Bousser MG, Rey A, et al. Effects of extraintracranial arterial bypass on cerebral blood flow and oxygen metabolism in humans. Stroke 1985;16:609-16.

19 Caplan LC, Schoene WC. Clinical features of subcortical arteriosclerotic encephalopathy (Binswanger's disease). Neurology 1978;28:1206-15.

20 Meyer JS, Kitagawa Y, Tanahashi N, et al. Evaluation of treatment of normal-pressure hydrocephalus. J Neurosurg 1985;62:513-21.

21 Shaw TG, Mortel KF, Meyer JS, et al. Cerebral blood flow changes in benign aging and cerebrovascular disease. Neurology 1984;34:855-62.

22 Meyer JS, Rogers RL, Mortel KF. Prospective analysis of longterm control of mild hypertension on cerebral blood flow. Stroke 1985;16:985-9.

23 Rogers RL, Meyer JS, Shaw TG, Mortel KF. Reductions in cerebral blood flow associated with chronic alcohol consumption. J Am Geriatr Soc 1985;31:540-3.

24 Meyer JS, Rogers RL, Mortel KF. Progressive cerebral ischaemia antedates cerebrovascular symptoms by two years. Ann Neurol 1984;16:305-13.

25 Rogers RL, Meyer JS, Mortel KF, Mahurin R. Decreased cerebral blood flow precedes multi-infarct dementia, but follows senile dementia of Alzheimer's type. Neurology 1986;36:1-6.

26 Rogers RL, Meyer JS, Shaw TG, et al. Cigarette smoking decreases cerebral blood flow suggesting increased risk for stroke. JAMA 1983;250:2796-800.

27 Meyer JS, Rogers RL, Mortel KF, Judd BW. Hyperlipidemia is a risk factor for reduced cerebral perfusion and stroke. Arch Neurol 1987;44:418-22.

28 Rogers RL, Meyer JS, Shaw TG, et al. The effects of chronic cigarette smoking on cerebrovascular responsiveness to $5 \%$ $\mathrm{CO}_{2}$ and $100 \% \mathrm{O}_{2}$ inhalation. $\mathrm{J}$ Am Geriatr Soc 1984; 32:415-20.

29 Rogers RL, Meyer JS, Judd BW, Mortel KF. Abstention from cigarette smoking improves cerebral perfusion among elderly chronic smokers. JAMA 1985;253:2970-4.

30 Ladurner G, Iliff LD, Sayer WD, Lechner H. In: A clinical approach to vascular (multi-infarct) dementia. Meyer JS, Lechner H, Reivich M (eds.): Cerebral Vascular Disease 4: Elsevier International Salzburg Conference, Amsterdam Excerpta Medica 1983, 236-43.

31 Alexander MP, Geschwind N: Dementia in the elderly, In: Albert ML (ed.): Clinical Neurology of Aging. New York, Oxford University Press, 1984, 254-76.

32 Whisnant JP, Cartlidge NEF, Elveback LR: Carotid and vertebral basilar transient ischaemic attacks: Effect of anticoagulants, hypertension, and cardiac disorders in survival and stroke occurrence, a population study. Ann Neurol 1978;3: 107-15.

33 Bougousslavsky J, Regli F, Van Melle G: Risk factors and concomitants of internal carotid artery occlusion or stenosis. Arch Neurol 1985;42:864-7.

34 Meyer JS, Charney JZ, Rivera M, Mathew NT: Cerebral embolization: prospective clinical analysis of 42 cases. Stroke 1971;2:541-4.

35 Rogers RL, Meyer JS, Mortel KF: additional predisposing risk factors for atherothrombotic cerebrovascular disease among treated hypertensive volunteers. Stroke 1987;18:335-41.

36 Weinberger J, Biscarra V, Weisberg MK, Jacobson JH: Factors contributing to stroke in patients with atherosclerotic disease of the great vessels: The role of diabetes. Stroke 1983;14: 709-12.

37 Meyer JS, Rogers RL, Mortel KF, Judd BW: Hyperlipidemia is a risk factor for decreased cerebral perfusion and stroke. Arch Neurol 1987;44:418-22.

38 Gotto A: Current concepts of hyperlipoproteinemia. In: Schettler G, Goto Y, Hata Y et al. (eds.): Atherosclerosis IV: Proceedings of the Fourth International Symposium. New York, Springer-Verlag NY, Inc., 1977, 209-19. 
39 Johnson PC, Doll SC, Cromey DW: Pathogenesis of diabetic neuropathy. Ann Neurol 1986;19:450-7.

40 Davis PH, Dambrosia JM, Schoenberg BS, et al.: Risk factors for ischemic stroke: a prospective study in Rochester, Minnesota. Ann Neurol 1987;22:319-27.

41 Rogers RL, Meyer JS, Shaw TG, et al.: Cigarette smoking decreases cerebral blood flow suggesting increased risk from stroke. JAMA 1983;250:2796-800.

42 Rogers RL, Meyer JS, Shaw TG, et al:: Reductions in regional cerebral blood flow associated with chronic consumption of alcohol. J Am Geriatr Soc 1983;31:540-3.

43 Obrist WD, Thompson HK, Wang HS, et al. Regional cerebral blood flow estimated by 133 Xenon inhalation. Stroke 1975;6: 245-56.

44 Meyer JS, Ishikawa N, Deshmukh VD, et al. Improved method for noninvasive measurement of regional cerebral blood flow by 133 Xenon inhalation. Stroke 1978;9:195-205.

45 Kitagawa Y, Meyer JS, Tachibana H. et al. CT-CBF correlates of cognitive deficits in multi-infarct dementia. Stroke 1984;15:1000-8.

46 O'Brien MD, Mallett BL. Cerebral perfusion rates in dementia.
J Neurol Neurosurg Psychiatry 1970;33:497-500.

47 O'Brien MD. Vascular disease and dementia in the elderly. In: Lynn-Smith W, Kinsborne M. (eds.) Aging and Dementia. New York: Spectrum 1977:77-90.

48 Meyer JS, Rogers RL, Judd BW, et al. Cognition and cerebral blood flow fluctuate together in multi-infarct dementia. Stroke, 1988, 163-9.

49 Roth M. Epidemiological studies. In: Alzheimer's Disease: Senile Dementia and Related Disorders (Aging, vol. 7) Katzman R, Terry RD, Bick AKL eds: New York, Raven Press 1978:337-9.

50 Schoenberg BS, Epidemiology of dementia due to cerebrovascular disease. In: Vascular or Multi-infarct Dementia. Meyer JS, Lechner H, Marshall J, Toole J (eds,): Mount Kisco, N.Y., Futura Publishing Co. 1988, 45-59.

51 Ueshima H, Takako O, Asakura S. Regional differences in stroke mortality and alcohol consumption in Japan. Stroke 1986; 7:19-24.

52 Wolf P, Kannel W. Controllable risk factors for stroke: preventive implications of trends in stroke mortality. In: Meyer JS, Shaw T, (eds.): Diagnosis and Management of Stroke and TIAs. London. Addison-Wesley Publishing Co.: 1982, 25-57.

\section{Moritz Heinrich Romberg and Ekbom's syndrome}

As a result of four papers, ${ }^{12}$ the well known syndrome of restless legs is justly credited to KA Ekbom Sr. His seminal paper ${ }^{1}$ referred to "asthenia crurum paraesthetica (Irritable Legs)". His second distinguished two forms: (1) "asthenia crurum paraesthetica" and (2) "asthenia crurum dolorosa"; he later ${ }^{2}$ settled for plain "restless legs syndrome". Others have referred to fidgety feet, and dyslysis. Spillane and colleagues described a similar condition of "painful legs and moving toes" relieved by sympathetic block with local anaesthetic.

Of historical interest is a passage in the English translation of the 2nd edition of Romberg's celebrated $A$ Manual of the Nervous Diseases of Man (1853). ${ }^{3}$ Romberg was a great nosologist, a devotee and translator of the works of Sir Charles Bell and Marshall Hall. He provided good descriptions of, inter alia: progressive muscular atrophy, carpal tunnel syndrome, neuralgic amyotrophy and in 1846 the Parry-Romberg syndrome of progressive hemifacial atrophy. Chapter 9 includes: "Anxietas tibiarum", a sense of painful restlessness in the lower extremities, especially in the legs and feet. The patient "did not know what to do with them. . though there was relief by change of position".

Though modern, symptomatic, empirical treatment is valuable, the aetiology remains an enigma.

\section{References}

1 Ekbom KA. Asthenia crurum paraesthetica ("Irritable Legs"). A new syndrome consisting of weakness, sensation of cold and nocturnal paraesthesia in the legs, responding to a certain extent to treatment in general. Acta Med Scand 1944;118:197-209.

2 Ekbom KA. Restless legs syndrome. Neurology 1960;10:868-73.

3 Romberg MH. A Manual of the Nervous Diseases of Man. 2 vols. Chapter 9: Hyperaesthesia of the Nerves of Muscular Sense. (Trans and ed Sieveking EH, London, The New Sydenham society, 1853. 